\title{
Editorial on robotics and artificial intelligence
}

\section{Introduction}

Artificial Intelligence (AI) in robotics indicates the use of a robot to model and imitate intelligent behavior. Research in Artificial Intelligence concentrates not only on the development but also its analysis of algorithms that learn to perform intelligent behavior with minimal human supervision. Artificial Intelligence has broad range; for instance in robotics, medical, e-commerce, gaming, diagnosis, military mathematics, planning and logistics. Artificial Intelligence in robotics is certainly the most interesting field in robotics. Ultimate Artificial Intelligence for robots would be hard as it is continuous process. It would be recreation of human thought process; a manmade machine which intellectual abilities same as human. This also includes the ability to learn about almost anything, the ability to reason, ability to use language and to formulate original ideas. But todays Artificial Intelligence have made progress but with limited AI. Few of them below are discussed about Robotics.

\section{Robots for autism therapy through "deep learning"}

Children with autism spectrum conditions often have difficulty in distinguishing emotional states of people around them. To remedy this problem, researchers from Massachusetts Institute of Technology (MIT) designed a kid friendly robot to demonstrate those emotions and to participate children in mimicking the emotions and responding to them in appropriate ways. They designed a new type of personalized machine learning that helps robots to evaluate the engagement and interest of each child in interactions, using data that are unique to that child. "Long term goal is actually not to create robots that will replace human therapist but to augment them with significant information that therapists can use to distinguish the therapy content and making them to engage in naturalistic interactions between robots and children with autism" was confirmed Oggi Rudovic, first author of the study.

\section{Quantum robotics}

Quantum mechanics assures to modernize the world of computers and communications by introducing algorithms which are much faster and more secure in transmitting information. Theoretical work has focused on using quantum computing to speed up with one of the most challenging aspects to resolve in information technology: machine learning which is used to design highly precise models and predictions. Scientist from Complutense University of Madrid (UCM) and University of Innsbruck (Austria) have studied which states that these tools can be used to apply to robots, automatons and other means that use Artificial Intelligence. They also demonstrated for the first time that quantum machines can respond the best and act fastest against the environment around them. More importantly, they adapt to the surrounding where the conventional ones are much slower and are unable to respond and learning process (Figure 1). ${ }^{1-4}$

\section{Robots can write meaningful news?}

Digitalization is the combination of digital technologies into our everyday life but it is also the progression of moving to a digital business. Media industry and news in specific is the initial point for the DPer News project, but almost virtually all businesses are moving

\author{
Volume 4 Issue 4 - 2018 \\ Manu Mitra \\ Department of Alumnus with Electrical Engineering, University \\ of Bridgeport, USA
}

Correspondence: Manu Mitra, Department of Alumnus with Electrical Engineering, University of Bridgeport, USA, Email mmitra@my.bridgeport.edu

Received: July 23, 2018 | Published: August I3, 2018

towards digitization. In some specific areas, robots can be used to support journalist by searching and examining data, but journalist still writes the story. In additional cases, robots could do the actual writing work. The DPer News project wants to find innovative method for robotilization that can assist the news companies to create more interesting news. Robots and computers are substituting people everywhere; pilots, doctors and even journalist. Keeping this in mind, scientist from Media Management and Transformation Centre (MMTC) at Jönköping International Business School launched the projects DPer (Digital Personalization of the News). ${ }^{6,7}$

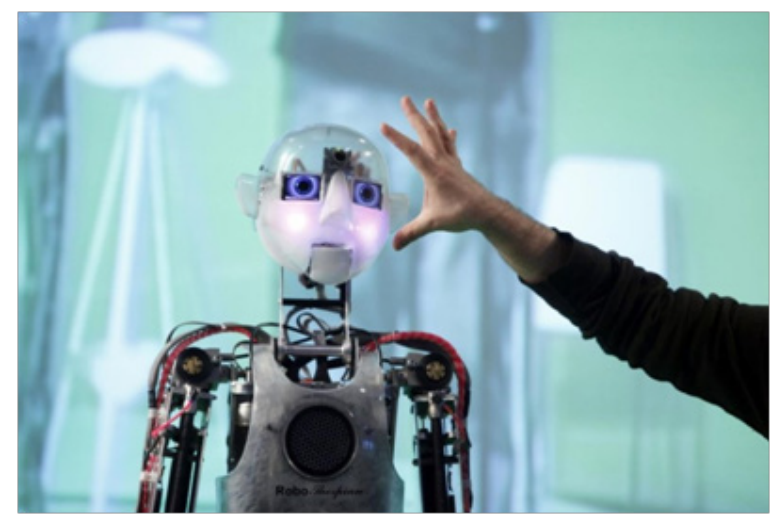

Figure I Illustrates theoretical effort on using quantum computing to advance machine learning. ${ }^{5}$ Image Credit: SINC.

\section{Merging, morphing and healing of mobile robots}

Various robots are controlled by robotic nervous systems in which sensors and actuators are associated to central processing unit. Nevertheless, in most of the cases robotic nervous systems are mapped firmly to the contour of the robot, which limits adaptability in their ability. Flexibility could be improved using modular robots that are made up of multiple units that can form collective bodies but orientation and control of modular robots have been forced by a limited set of predefined forms that the units can change into. Scientist at the Université libre de Bruxelles designed a self-configurable modular robots that can split, merge and even self-heal while retaining full sensorimotor control. This work may take us nearer to creating robots that can autonomously change their shape, size and function (Figure 2). ${ }^{9}$ 


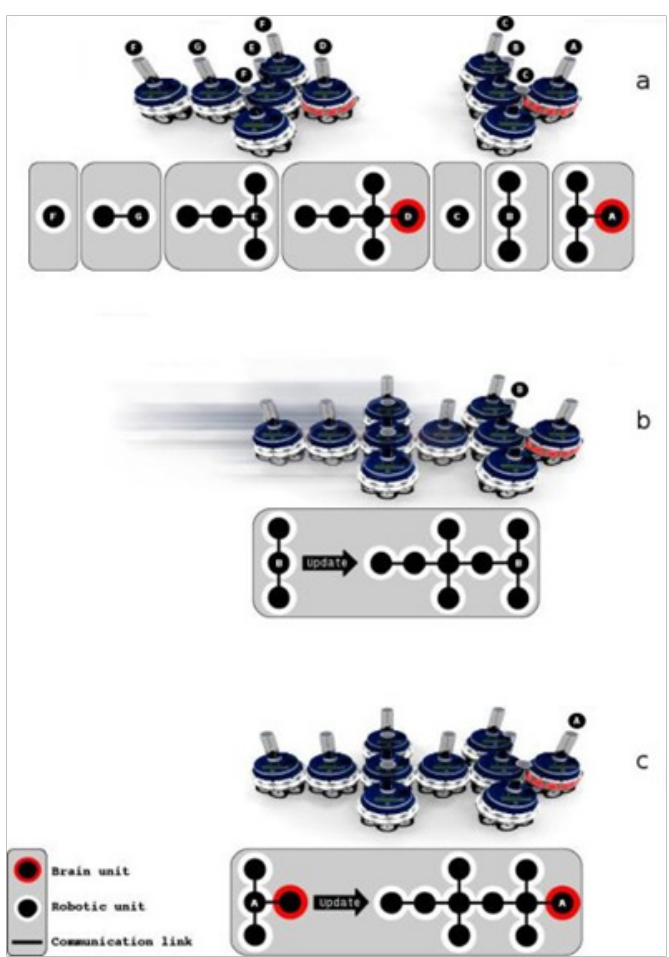

Figure 2 Illustrates a self-reconfiguring modular robots prototype. ${ }^{8}$ Image Credit: Iridia Lab ULB.

\section{Robotic fish that can mimic and watch back live fish}

Porfiri and his team members tapped advances in real time tracking software, designed custom robotic fish and tested the first closed loop control system presenting a bio inspired robotic model communicating in three dimension with live zebra fish. They tested robotic model under several various experimental conditions, but in most of the cases, the model and the live fish were separated by transparent panel. In inclination tests, zebra fish showed great affinity and no signs of fear or anxiety toward the robotic fish that mirrored its own actions rather than a robot that followed a pre-set pattern. For more than a decade, bio mimetic robots have been deployed along side with live fish to better understand the animal behavior including leadership, social cues and fear. This experiment was conducted by Prof. Maurizio Porfiri professor of mechanical and aerospace engineering at New York University Tandon School of Engineering. To complete, International Robotics \& Automation Journal (IRATJ) ISSN: 25748092 will be devoted to move forward with the international research community to attain robust possible scientific picture on coming up extent forum parallel equality for betterment of human mankind (Figure 3).

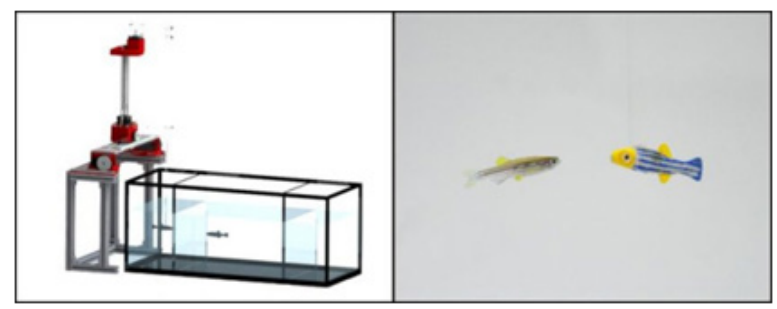

Figure 3 Illustrates Robotic fish with zebra fish.The robotic system allows the robotic mimic to both see and replicate the behavior of live zebrafish in real time. ${ }^{10}$ Image Credit: Image courtesy of NYU Tandon School of Engineering.

\section{Acknowledgements}

None.

\section{Conflict of interest}

The author declares there is no conflict of interest.

\section{References}

1. Robotics and Artificial Intelligence. Computer Science \& Engineering, University of Minnesota; 2018

2. Harris T. How robots work. 2002

3. Personalized 'deep learning' equips robots for autism therapy: Machine learning network offers personalized estimates of children's behavior. Massachusetts Institute of Technology; Science Daily. 2018

4. Giuseppe Davide Paparo, Vedran Dunjko, Adi Makmal, et al. Quantum speedup for active learning agents. Physical Review X. 2014;4(3):1-14.

5. Pressing the accelerator on quantum robotics. Plataforma SINC; Science Daily. 2014.

6. Can robots write meaningful news? Jönköping University, Science Daily; 2017.

7. Nithin Mathews, Anders Lyhne Christensen, Rehan O'Grady, et al. Mergeable nervous systems for robots. Nature Communications. 2017;8(1).

8. AI -- Engineering: merging, morphing, mobile robots: Self-reconfiguring modular robots that can merge, split and even self-heal while retaining full sensori motor control. Université libre de Bruxelles, Science Daily; 2017.

9. Changsu Kim, Tommaso Ruberto, Paul Phamduy, et al. Closed-loop control of zebrafish behaviour in three dimensions using a robotic stimulus. Scientific Reports. 2018;8(1).

10. Robotic fish can 'see' and mimic live fish. NYU Tandon School of Engineering, Science Daily; 2018. 